\title{
Haemoptysis in adults: a 5-year study using the French nationwide hospital administrative database
}

\author{
Caroline Abdulmalak ${ }^{1}$, Jonathan Cottenet $^{2}$, Guillaume Beltramo ${ }^{1}$ \\ Marjolaine Georges ${ }^{1,3}$, Philippe Camus ${ }^{1,3,4}$, Philippe Bonniaud ${ }^{1,3,4,5}$ and \\ Catherine Quantin $2,3,4,5$
}

Affiliations: 'Service de Pneumologie et Soins Intensifs Respiratoires, Centre Hospitalier Universitaire du Bocage, Dijon, France. ${ }^{2}$ Department of Medical Informatics, University Hospital, Dijon, France. ${ }^{3}$ Faculty of Medicine and Pharmacy, University of Burgundy, Dijon, France. ${ }^{4}$ INSERM U866, Dijon, France. ${ }^{5}$ Both authors contributed equally.

Correspondence: Philippe Bonniaud, Service de Pneumologie et Soins Intensifs Respiratoires, Centre Hospitalier Universitaire du Bocage, 21079, Dijon, France. E-mail: philippe.bonniaudachu-dijon.fr

ABSTRACT Haemoptysis is a serious symptom with various aetiologies. Our aim was to define the aetiologies, outcomes and associations with lung cancer in the entire population of a high-income country.

This retrospective multicentre study was based on the French nationwide hospital medical information database collected over 5 years (2008-2012). We analysed haemoptysis incidence, aetiologies, geographical and seasonal distribution and mortality. We studied recurrence, association with lung cancer and mortality in a 3-year follow-up analysis.

Each year, 15000 adult patients (mean age 62 years, male/female ratio 2/1) were admitted for haemoptysis or had haemoptysis as a complication of their hospital stay, representing $0.2 \%$ of all hospitalised patients. Haemoptysis was cryptogenic in 50\% of cases. The main aetiologies were respiratory infections $(22 \%)$, lung cancer $(17.4 \%)$, bronchiectasis $(6.8 \%)$, pulmonary oedema $(4.2 \%)$, anticoagulants (3.5\%), tuberculosis (2.7\%), pulmonary embolism (2.6\%) and aspergillosis (1.1\%). Among incident cases, the 3 -year recurrence rate was $16.3 \%$. Of the initial cryptogenic haemoptysis patients, $4 \%$ were diagnosed with lung cancer within 3 years. Mortality rates during the first stay and at 1 and 3 years were 9.2\%, 21.6\% and $27 \%$, respectively.

This is the first epidemiological study analysing haemoptysis and its outcomes in an entire population. Haemoptysis is a life-threatening symptom unveiling potentially life-threatening underlying conditions.

@ERSpublications

Haemoptysis is ominous: there is often no clear aetiology and $4 \%$ of patients develop lung cancer during follow-up http://ow.ly/KqJDG 


\section{Introduction}

Haemoptysis is a symptom varying in its seriousness, and in most cases hospitalisation is required. A number of aetiologies are described in the literature. Prevalence differs depending on when the study was performed and the country of the study [1-8]. To the best of our knowledge, epidemiological studies conducted in high-income countries are old single-centre studies involving $\sim 100$ patients. In a retrospective study in Israel [5], the main aetiologies were infectious respiratory disease (34\%), followed by bronchiectasis (20\%) and lung cancer (19\%). In a study from South Korea [1], bronchiectasis was the main cause of haemoptysis (32.6\% of cases) followed by tuberculosis (18.5\%) and pulmonary fungal infections (10.8\%). A French retrospective study that included $>6000$ patients [4] found that bronchial carcinoma accounted for $14.9 \%$ of cases and was the leading cause of haemoptysis.

Haemoptysis of unknown origin, also called cryptogenic haemoptysis, is a critical issue as it accounts for $3-42 \%$ of all haemoptysis cases $[4,5]$. The challenges of cryptogenic haemoptysis include missing a serious underlying condition such as lung cancer and the need to prevent the risk of recurrence if the initial cause is not correctly identified and treated. In a retrospective study, $6 \%$ of 135 patients [9] developed bronchial carcinoma during the follow-up of cryptogenic haemoptysis. In contrast, in a French prospective study of 81 patients with cryptogenic haemoptysis [2], no increased risk of lung malignancy was found.

The objectives of our study were to provide an epidemiological update on haemoptysis in France, to assess outcomes in patients presenting with this symptom and to determine the benefit of close follow-up and extended investigations when first-line examinations are normal. The French national administrative database, also called the medicalisation of information system programme (PMSI) provides a huge amount of epidemiological information concerning hospitalised French patients [10-16]. Data pertaining to haemoptysis are reliable enough to count such patients and to describe the aetiologies of the condition.

\section{Material and methods}

The national administrative database: PMSI

Inspired by the American diagnosis-related groups model, the PMSI has gathered national administrative health data in France since 1991. The system was extended to all French healthcare facilities in 1997. The PMSI was initially designed to analyse the activities of hospitals and to contribute to the elaboration of strategic healthcare plans. Since 2008, each hospital's budget has depended on the medical activity described in a specific programme which compiles discharge abstracts related to all admissions. Information in these abstracts is anonymous and covers both medical and administrative data. Diagnoses identified during hospital stays are coded according to the tenth edition of the International Classification of Diseases (ICD-10). Each facility produces its own standardised anonymous dataset which are then compiled at the national level. The fact that these national data are used to allocate hospital budgets has encouraged improvements in data quality in terms of coherence, accuracy and exhaustiveness.

\section{Population}

This study was a retrospective multicentre study based on nationwide PMSI data collected between January 2008 and December 2012. The use of the PMSI for this study was approved by the National Commission for Data Protection (CNIL 1576793). We included all patients aged $>18$ years who were admitted to hospital with haemoptysis during this period. All hospitalisations whose main or related or associated diagnosis was haemoptysis (ICD-10 code R042) were included. From the PMSI database, we collected data about age, sex, area of residence, reason for admission to hospital, related or associated diagnoses, month of discharge, length of stay, use of therapeutic embolisation, number of recurrences, time to recurrence and in-hospital mortality. We recorded data about the prevalence and incidence of haemoptysis in France. Prevalence corresponded to the number of patients presenting with haemoptysis during a period (recurrences during this period were excluded). Incidence was defined as the number of new cases per year. In the present work, we assumed that patients had not had haemoptysis during the previous year only. In fact, we were unable to identify events prior to the previous year. Prevalence and incidence data for each aetiological group (see below) were analysed.

\section{Variables}

In the PMSI coding system, the clinician must indicate the main diagnosis (symptom or disease) and any associated diagnoses (for conditions that require substantial levels of care). The related diagnosis could complete the main diagnosis when it corresponded to a Z-code (follow-up, treatments such as chemotherapy) and when a chronic disease could not be recorded during the hospitalisation. We therefore determined the potential aetiologies of haemoptysis when the diagnosis reported was the main, related or associated diagnosis. The aetiologies recorded were active pulmonary tuberculosis, pulmonary oedema, respiratory infection, mitral stenosis, bronchopulmonary carcinoma, aspergillosis, pulmonary embolism, systemic disease (Goodpasture syndrome, micropolyangiitis, granulomatosis with polyangiitis and lupus), 
the adverse effects of anticoagulants, bronchiectasis and cystic fibrosis, pulmonary haemosiderosis, chest trauma, airway foreign body, bronchial endometriosis, bronchial benign tumour and vascular malformation (fistula and aneurysm). A detailed list of the items that were recorded is available in online supplementary table S1.

We studied the geographical distribution of haemoptysis in France to determine whether there were regional differences, and the seasonal distribution of haemoptysis. We studied long-term outcomes in patients with respect to recurrence, therapeutic embolisation and hospital mortality during 3 years of follow-up for patients admitted in 2008 and 2009, and 1 year of follow-up for patients admitted from 2008 to 2011. Recurrence was analysed according to the aetiology of the haemoptysis and the use of embolisation. Finally, all incident cases with none of the above aetiologies coded as the main/associated/ related diagnosis were recorded as cryptogenic haemoptysis. The frequency of the emergence of a disease (lung cancer, vasculitis or vascular malformation) was studied.

\section{Statistical analysis}

For bivariate analyses of qualitative variables, we used the Chi-squared test to compare percentages. Mean values of quantitative variables were compared using linear regressions. p-values $<0.05$ were considered to be statistically significant. SAS 9.2 software (SAS Institute Inc., Cary, NC, USA) was used for analysis.

\section{Results}

Population

During the period January 2008 to December 2012, 10 million patients were hospitalised yearly in French public and private hospitals and were registered in the national administrative database (table 1). Among these patients, 15000 (range 14559-16713) were admitted each year for haemoptysis or had haemoptysis as a complication of their stay. This represents $0.2 \%$ of all patients hospitalised in France during the period. This proportion was relatively constant over time. In our study, the majority of patients hospitalised for (or with) haemoptysis were men. The mean age of this population was 62 years. The results for the prevalence and incidence of haemoptysis with regard to mean age and sex ratio were superimposable (table 1). The mean duration of the initial hospital stay for haemoptysis was $8.0 \pm 12.5$ days, and during this stay $9.0 \%$ of these patients were admitted to an intensive care unit (7-10\%). One-third of the patients had a computed tomography scan. Almost half of the patients underwent an endobronchial intervention, but the PMSI database did not distinguish between diagnostic and interventional procedures (online supplementary table S2). During their hospital stay, 1\% of the patients underwent thoracic surgery, but we do not know if the surgery was performed to treat the haemoptysis or if this symptom was a consequence of the surgery (online supplementary table S2).

\section{Seasonal variation}

The incidence of haemoptysis peaked in March (9.5\% of annual hospitalisations for haemoptysis) and in October $(8.5 \%)$ of each year. Conversely, we observed a significant relative decrease in the incidence of haemoptysis in August $(6.7 \%, \mathrm{p}<0.01)$. The seasonal profiles for the years of the study were perfectly superimposable (fig. 1).

Haemoptysis thought to be related to an infection followed the same seasonality (online supplementary fig. S1), whereas seasonal variations for other causes of haemoptysis showed no significant trends.

\begin{tabular}{|c|c|c|c|c|c|}
\hline & 2012 & 2011 & 2010 & 2009 & 2008 \\
\hline Patients n & 9702549 & 9571372 & 9440401 & 9416344 & 9154441 \\
\hline Prevalence & $17485(0.2)$ & $16526(0.2)$ & $15960(0.2)$ & $16360(0.2)$ & 15241 (0.2) \\
\hline Age years & $63 \pm 18$ & $63 \pm 18$ & $62 \pm 18$ & $62 \pm 18$ & $62 \pm 18$ \\
\hline Male \% & 68.9 & 68.8 & 69.9 & 69.1 & 69.5 \\
\hline Incidence & $16713(0.2)$ & $15818(0.2)$ & $15196(0.2)$ & $15666(0.2)$ & $14559(0.2)$ \\
\hline Age years & $63 \pm 18$ & $62 \pm 18$ & $62 \pm 18$ & $62 \pm 18$ & $62 \pm 17$ \\
\hline Male \% & 68.9 & 68.7 & 69.9 & 69.0 & 69.5 \\
\hline
\end{tabular}

Data are presented as mean \pm SD or $\mathrm{n}(\%)$, unless otherwise stated. 


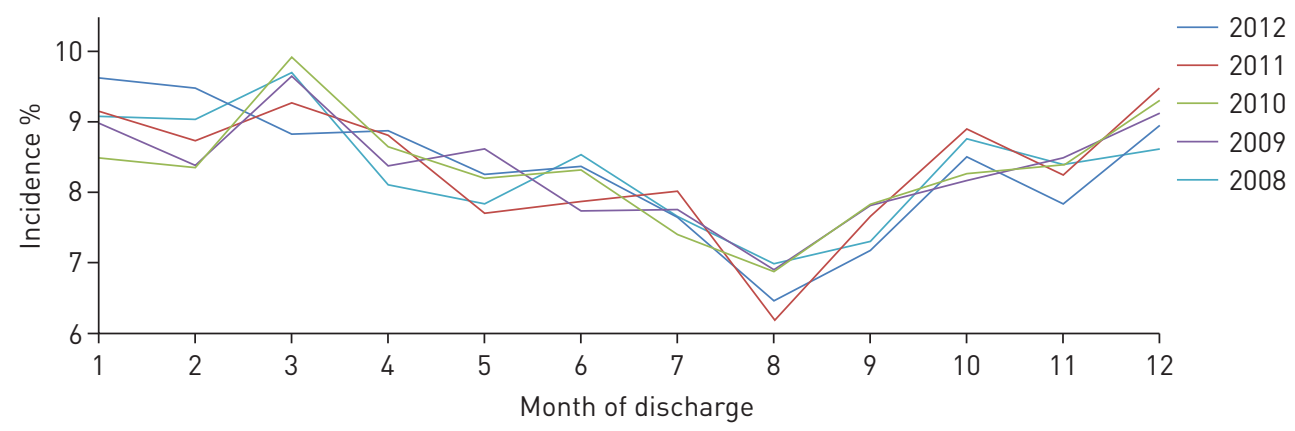

FIGURE 1 Seasonal variations in the incidence of haemoptysis. Incidence of haemoptysis according to the month of discharge between 2008 and 2012.

\section{Aetiological distribution}

Haemoptysis of unknown origin accounted for the majority of incident cases (table 2). Respiratory infections, including viral, bacterial and fungal pneumonia and bronchitis, but not aspergillosis and active tuberculosis were the most frequent aetiologies of haemoptysis (22\%), and this was stable over the 5 years of our analysis. Neoplastic pulmonary aetiologies included primary lung cancer and metastasis from the lung or other sites. This group constituted the second most frequent aetiology of haemoptysis, accounting for $17.4 \%$ of all incident cases.

\section{Geographical distribution}

The territorial distribution of the incidence of haemoptysis in 2012 was collected according to the patient's department of residence. The incidence of haemoptysis was highest in areas with the highest population density (fig. 2). The geographical distribution of haemoptysis and haemoptysis due to tuberculosis in 2012 revealed major territorial disparities, with the highest incidence of haemoptysis (2.5-4.1\%) in the French departments of Paris, Bouches du Rhône, Nord, Seine-Saint-Denis and Pas-de-Calais (fig. 2 and online supplementary fig. S2). These observations were similar in 2008.

\section{Follow-up at 1 and 3 years}

\section{Recurrence}

Of the incident cases in 2008 and 2009, 16.6\% and 16.1\% of patients, respectively, experienced recurrence during the 3 years of follow-up (table 3). The mean number of recurrences was 1.5 within the 3 years and the mean time to recurrence was 154 days. Cryptogenic haemoptysis, bronchopulmonary cancer and

\begin{tabular}{|c|c|c|c|c|c|}
\hline Associated diagnosis ${ }^{\#}$ & 2012 & 2011 & 2010 & 2009 & 2008 \\
\hline Cryptogenic haemoptysis & 48.9 & 49.4 & 50.0 & 51.0 & 52.0 \\
\hline Respiratory infections & 23.1 & 22.4 & 21.8 & 22.1 & 20.6 \\
\hline Bronchopulmonary cancer & 17.8 & 18.0 & 17.8 & 16.7 & 16.9 \\
\hline Anticoagulant treatment & 9.5 & 8.3 & 7.4 & 7.2 & 5.7 \\
\hline Bronchiectasis/cystic fibrosis & 6.9 & 7.0 & 6.5 & 6.8 & 6.8 \\
\hline Pulmonary oedema/mitral stenosis & 4.6 & 4.1 & 4.1 & 4.1 & 3.6 \\
\hline Anticoagulant accident & 4.0 & 3.7 & 3.3 & 3.0 & 2.3 \\
\hline Pulmonary embolism & 2.8 & 2.6 & 2.5 & 2.4 & 2.4 \\
\hline Lung tuberculosis & 2.5 & 2.7 & 2.9 & 3.0 & 3.4 \\
\hline Aspergillosis & 1.2 & 1.3 & 1.0 & 0.8 & 0.9 \\
\hline Thoracic trauma & 0.7 & 0.8 & 0.7 & 0.6 & 0.5 \\
\hline Systemic disease & 0.2 & 0.2 & 0.2 & 0.2 & 0.2 \\
\hline Vascular malformation & 0.2 & 0.1 & 0.2 & 0.2 & 0.2 \\
\hline Bronchial benign tumour & 0.1 & 0.2 & 0.2 & 0.2 & 0.2 \\
\hline Tracheal/bronchial foreign body & 0.1 & 0.1 & 0.1 & 0.1 & 0.1 \\
\hline Pulmonary haemosiderosis & 0.1 & 0.1 & 0.1 & 0.1 & 0.1 \\
\hline Bronchial endometriosis & 0.01 & 0.01 & 0.01 & 0.02 & 0.01 \\
\hline
\end{tabular}

\footnotetext{
\#: two diagnoses may be associated.
} 
FIGURE 2 Geographical distribution of haemoptysis in 2012. The territorial distribution of haemoptysis incidence in 2012 was collected according to each patient's department of residence. Haemoptysis was higher in areas of high population density.

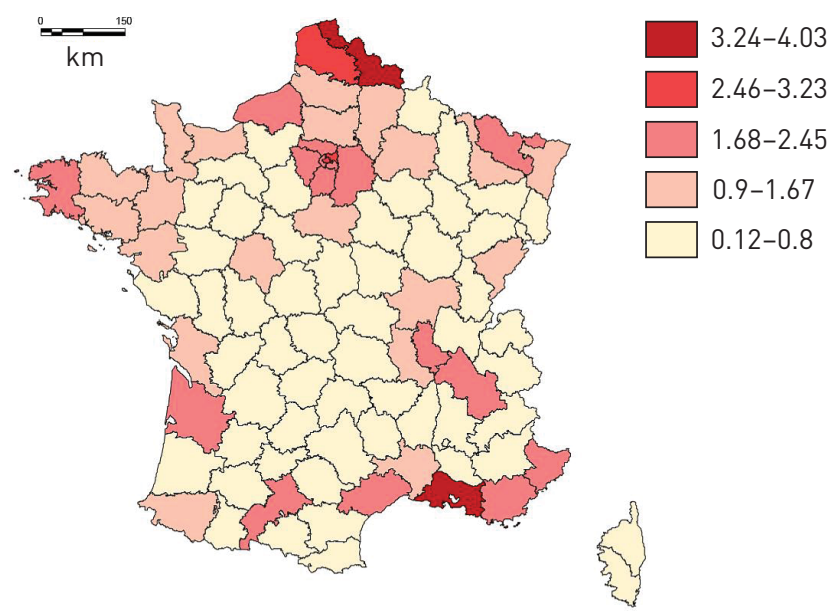

respiratory infections were the most common aetiologies found at recurrence. The distribution of aetiologies during recurrences was similar in 2008 and 2009 (fig. 3).

\section{New diagnoses}

Among haemoptysis cases recorded in 2009 as "unknown origin" and "respiratory infections", 9.6\% and $10.4 \%$, respectively, were subsequently coded for bronchopulmonary cancer during the 3 years of follow-up (table 4). The results were almost identical in 2008 (online supplementary table S3). Time to the diagnosis of lung cancer for haemoptysis of "unknown origin" and "haemoptysis due to respiratory infections" is provided in online supplementary figures S3 and S4. Of the haemoptysis cases initially classified as cryptogenic, $8.9 \%$ recurred with a diagnosis of bronchiectasis, and $3.3 \%$ recurred with a diagnosis of active tuberculosis. In contrast, $47.7 \%$ of haemoptysis cases initially recorded as "respiratory infections" recurred with the same aetiology, while 3.1\% of recurrences following an initial aetiology of "respiratory infections" were diagnosed as "active tuberculosis" (online supplementary table S4). Of note, lung cancer was diagnosed in $12 \%$ of the recurrences following an initial diagnosis of respiratory infection.

\section{Use of embolisation}

Embolisation was used in $2.4 \%$ of the first episodes of haemoptysis. Among these cases, 572 (30\%) were recorded as "haemoptysis of unknown origin", 509 (26.8\%) were associated with lung cancer and 409 (21.5\%) were related to respiratory infection.

During the 3 years of follow-up, $4.1 \%$ and $4.3 \%$ of incident cases in 2008 and 2009, respectively, required embolisation either for the first episode or during recurrences. Among patients who required the procedure, the mean \pm SD number of embolisations was $1.1 \pm 0.5$.

\section{Hospital mortality}

In 2008, in-hospital mortality for the first episode of haemoptysis was $8.7 \%$. The percentage increased during the following years, reaching $10.1 \%$ in 2011 . Hospital mortality, excluding deaths due to lung cancer, followed the same trend, increasing from $5.9 \%$ to $7.1 \%$. For incident cases in 2011, the 1-year mortality rate, excluding deaths from lung cancer, was $15.7 \%$. For incident cases in 2009, the 3-year mortality rate was $20 \%$. The 1 -year mortality rate increased significantly with time $(p<0.01)$ (table 5).

TABLE 3 Recurrence of haemoptysis during 3 years of follow-up

\begin{tabular}{lcc} 
& $\mathbf{2 0 0 9}$ & $\mathbf{2 0 0 8}$ \\
\hline Incident cases & 15666 & 14559 \\
Recurrence \% & 16.1 & 16.6 \\
Average number of recurrences & 1.5 & 1.5 \\
Time to recurrence days & $153 \pm 242$ & $155 \pm 245$
\end{tabular}

Data are presented as $\mathrm{n}$ or mean $\pm \mathrm{SD}$, unless otherwise stated. 


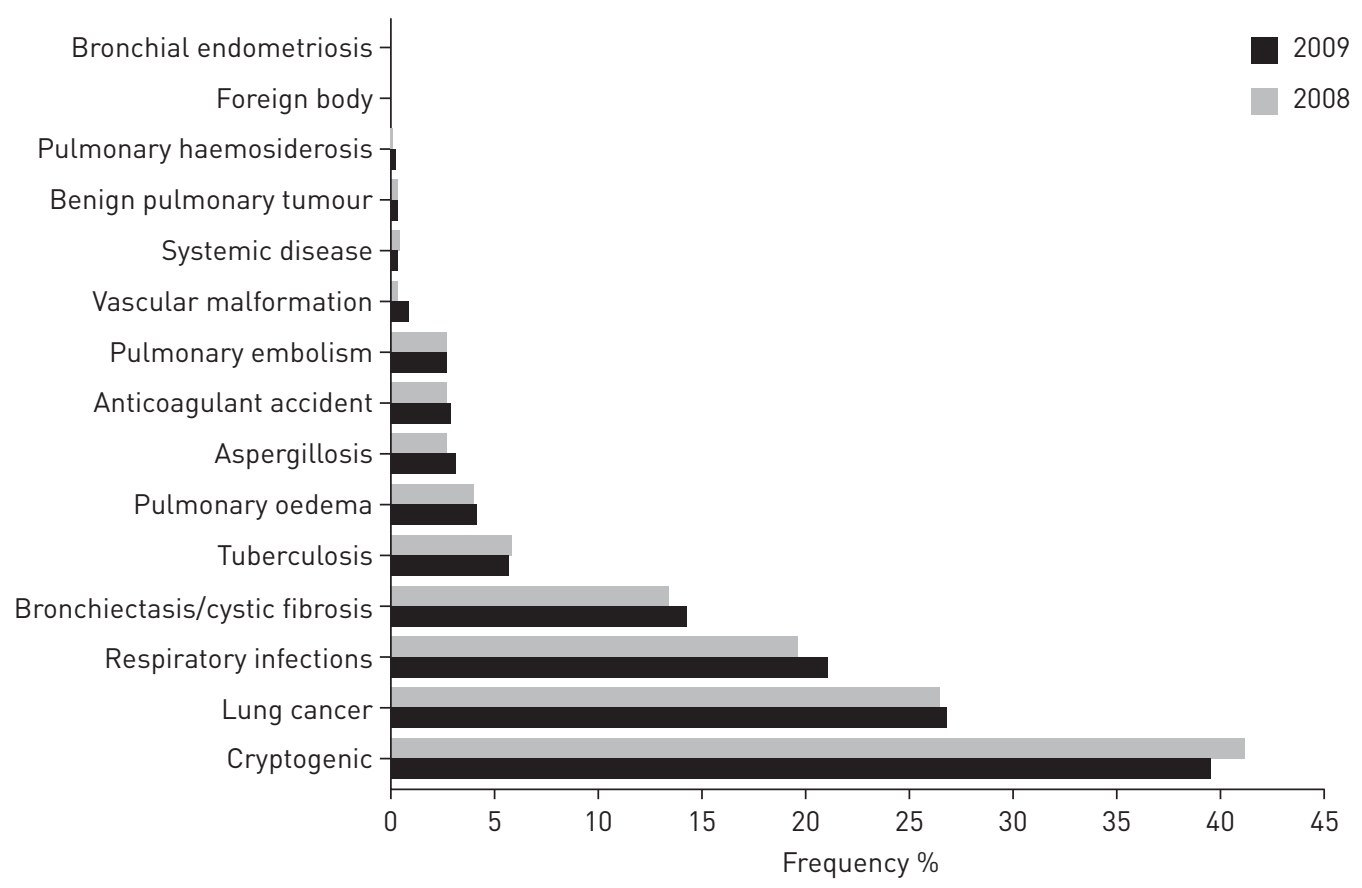

FIGURE 3 Recurrence of haemoptysis diagnosed in 2008 and 2009. All recurrences during the 3 years of follow-up were analysed. The first identified cause was lung cancer, followed by respiratory infections and bronchiectasis/cystic fibrosis.

\section{Discussion}

This 5-year retrospective study is, to our knowledge, the first to analyse haemoptysis, its aetiologies and outcomes in the population of a whole country, France. We found that haemoptysis accounted for $0.2 \%$ of hospitalised patients. In this high-income country, we showed that the most frequent aetiologies were respiratory infections, lung cancer and bronchiectasis. We also demonstrated that haemoptysis of unknown origin accounted for a large percentage of cases. A significant proportion of these were associated with the development of lung cancer during follow-up. Finally, haemoptysis is a symptom that portends a bleak outcome, as the 3 -year mortality rate was $20 \%$.

The main limitation of the present study was the coding system, which may have induced some inaccuracies. The results may have been biased by coding mistakes or omissions, which may have led to the under- or overestimation of both incidence and aetiologies. Moreover, the code for haemoptysis may have been recorded more often in cases requiring substantial amounts of care and as a result we may have over-selected serious cases of haemoptysis. The initial high percentage (almost 50\%) of cryptogenic haemoptysis may also be overestimated, and may result from inaccurate coding. However, as the frequency of haemoptysis found in our study correlates with previous studies, we feel that such inaccuracies may not be considerable. Indeed, recent studies that investigated the reliability of PMSI data by comparing them with reference data from other sources, including a stroke registry, a perinatal survey and vaccine safety assessments, showed that PMSI data can be reliably used for epidemiological purposes [10-13].

In the present work, the incidence of haemoptysis varied during the year and confirmed the results of other studies, which found two peaks, one in March and one in October [4]. Seasonal variations in the incidence of vascular diseases and overall mortality have already been demonstrated [17-20]. The main hypothesis to explain these very reproducible variations is the infectious origin of a high percentage of haemoptysis cases.

TABLE 4 Frequency of new diagnoses during the 3 years of follow-up according to initial diagnosis in 2009

\begin{tabular}{ccccccc}
$\begin{array}{c}\text { Bronchopulmonary } \\
\text { cancer }\end{array}$ & Tuberculosis & $\begin{array}{c}\text { Systemic } \\
\text { disease }\end{array}$ & $\begin{array}{c}\text { Vascular } \\
\text { malformation }\end{array}$ & Aspergillosis & $\begin{array}{c}\text { Bronchiectasis/ } \\
\text { cystic fibrosis }\end{array}$ & Anticoagulants \\
\hline 10.1 & 1.5 & 0.1 & 0.4 & 0.6 & 2.8 & 5.6 \\
10.4 & 2.3 & 0.3 & 0.1 & 0.9 & 6.6 & 10.4
\end{tabular}

Data are presented as \%. 
TABLE 5 Mortality rates at 1 and 3 years

2011

10.1

7.1

23.3

$69 \pm 97,17(0-109)$

15.7

1-year mortality rate (excluding lung cancer)

Survival days

3-year mortality rate Survival days

3-year mortality rate (excluding lung cancer) Survival days

\section{Cryptogenic haemoptysis}

Mortality at initial stay

1 -year mortality rate

Survival days

3 -year mortality rate Survival days
$68 \pm 97,10(0-108) \quad 75 \pm 104,16.5(0-124) \quad 74 \pm 101,19(0-124.5)$

27.2

$204 \pm 278,64(0-317)$

20.1

$243 \pm 303,91(0-426)$

5.3

13.7

$69 \pm 95,16(0-112) \quad 72 \pm 101,17(0-120)$
5.6
2009

Data are presented as $\%$ or mean \pm SD and median (interquartile range). Mortality rates describe in-hospital mortality only.

Other hypotheses, such as hypertensive crises due to cold weather, variations in metabolism, the intake of vitamin $\mathrm{C}$ and variations in atmospheric pressure have been put forward [17-20].

Mortality rates in our study were substantial. Our results concerning in-hospital mortality (7\% when deaths from lung cancer were excluded) during the first episode of haemoptysis did not differ from those in other studies $[1,5,21]$. The mortality rates in the subgroup of haemoptysis of unknown origin were similar, suggesting that the lack of a described aetiology at the initial evaluation was not a distinct risk factor for increased mortality in our study. However, some deaths could have been due to lung cancer. Nonetheless, $20 \%$ of patients, with the exclusion of lung cancer patients, died during the 3 years of follow-up. These results are probably underestimated because the studied mortality concerned in-hospital mortality only. Few studies have focused on the follow-up of patients with haemoptysis. Among these, one French prospective study of 39 chronic obstructive pulmonary disease (COPD) patients presenting with cryptogenic haemoptysis found a mortality rate of $2.6 \%$ at 5 years [22]. A North American retrospective study of patients with cryptogenic haemoptysis found a 5-year mortality rate of $9.1 \%$ [23]. The observed differences are probably due to the small number of highly selected patients in the former study [22], which may have led to an underestimation of overall mortality. HerTH et al.[9] found a mortality rate of $13 \%$ after 6 years. All of the patients in the Herth et al. study died from the evolution of underlying diseases such as COPD, lung cancer, cirrhosis and granulomatous polyangiitis. Although we suspect that mortality in our study may have been related to underlying conditions rather than haemoptysis per se, the PMSI database did not allow us to make such distinctions.

In the present work, lower respiratory tract infection was the main cause of haemoptysis. These results tally with a retrospective study from Israel [5], although the proportion of respiratory infections was lower in our study ( $34 \%$ versus $23 \%$, respectively). However, both studies suffer from the lack of an accurate definition of respiratory infections. In our study, clinicians were free to code "respiratory infections" in the absence of radiological documentation or bacteriological proof, which may have overestimated their incidence.

Pulmonary cancers were the second most frequent cause of haemoptysis, with an average incidence of 17.4\%. These results are in keeping with the study by Hirshberg et al. [5] (19\%), but differ from other studies. In a North American study, lung cancer accounted for $29 \%$ of cases [6]. In a South Korean study, LEE et al. [1] found lung cancers in $5.9 \%$ of their population of haemoptysis patients. These differences may be due to the higher incidence of tuberculosis, and therefore bronchiectasis, in Asia.

Haemoptysis as an adverse effect of anticoagulant therapy represented $4 \%$ of all haemoptysis cases in our study. As the codes for anticoagulants do not engender modifications in the budget allocated for the hospitalisation, this frequency may be underestimated. This rate is high compared with the results of other studies, although in many studies, this aetiology was not mentioned or analysed. LeE et al. [1] focused on haemoptysis in patients with bronchiectasis and found that anticoagulant and antiplatelet therapy did not increase the risk of bleeding in this population, whereas SAVALE et al. [2] found treatments predisposing to 
haemorrhage in $24 \%$ of their patients. It is difficult to determine whether such treatments are the real cause or a risk factor of haemoptysis. It would be interesting to know what treatments are associated with haemoptysis and, specifically, whether new anticoagulation treatments are more often associated with the symptom. The main adverse effects reported so far include gastrointestinal, cerebral and pericardial haemorrhage [24], but no studies have shown an increased risk of haemoptysis with these new treatments $[24,25]$. Unfortunately, we were not able to study any association between the risk of haemoptysis and specific anticoagulant treatments, as the PMSI database does not allow the identification of the type of anticoagulant therapy used. There is no specific code for antiplatelet treatment in the PMSI database. Moreover, a number of cases of haemoptysis associated with anticoagulant or antiplatelet therapy were probably associated with the underlying pathologies and should be analysed with caution, particularly when haemoptysis recurs.

During follow-up in our study, $42.1 \%$ of recurrences were recorded as cryptogenic, and in $26.6 \%$ of these the recurrences were ultimately related to lung cancer. This could be explained by the high frequency of recurrent haemoptysis in lung cancer $[26,27]$.

Among patients with cryptogenic haemoptysis, 9.6\% were diagnosed with lung cancer within the 3-year period of follow-up. However, we suspect that in a number of cases the initial haemoptysis was not cryptogenic and that clinicians were waiting for the results of pathology examinations before coding for lung cancer. In the literature, the mean delay between the first symptoms and the pathology results confirming lung cancer is between 20 days and 4 weeks $[28,29]$. We therefore considered that lung cancer was the aetiology of the initial haemoptysis episode only when it was diagnosed within 2 months of the initial haemoptysis. In fact, a large proportion of these patients were diagnosed within 2 months of hospitalisation for haemoptysis, while another $4 \%$ of patients with cryptogenic haemoptysis were subsequently diagnosed with lung cancer during the long-term follow-up. Few studies have focused on the onset of lung cancer during the follow-up of patients with cryptogenic haemoptysis. In their retrospective study, Herth et al. [9] found that 6\% of patients with cryptogenic haemoptysis developed lung cancer during the 3 years of follow-up. The rate increased to $10 \%$ in the subgroup of smokers aged $>40$ years.

In conclusion, ours is the first study to analyse the incidence and outcome of haemoptysis in the whole population of a country. Our results show that haemoptysis is still an ominous symptom. Indeed, a large proportion of haemoptysis cases had no clear aetiology and $4 \%$ were subsequently diagnosed with lung cancer during follow-up. Due to this significant incidence of lung cancer diagnosed during follow-up, we believe that there is a need for a dedicated follow-up of these patients to detect cancer at an earlier stage. The 3-year in-hospital mortality rate, excluding lung cancers, was very high (20\%), and was probably more closely related to the underlying pathology than to haemoptysis itself.

Acknowledgements

We thank Philip Bastable (Research Unit, Dijon University Hospital, Dijon, France) for correcting the manuscript.

\section{References}

1 Lee BR, Yu JY, Ban HJ, et al. Analysis of patients with hemoptysis in a tertiary referral hospital. Tuberc Respir Dis 2012; 73: 107-114.

2 Savale L, Parrot A, Khalil A, et al. Cryptogenic hemoptysis: from a benign to a life-threatening pathologic vascular condition. Am J Respir Crit Care Med 2007; 175: 1181-1185.

3 Abal AT, Nair PC, Cherian J. Haemoptysis: aetiology, evaluation and outcome - a prospective study in a third-world country. Respir Med 2001; 95: 548-552.

4 Boulay F, Berthier F, Sisteron O, et al. Seasonal variation in cryptogenic and noncryptogenic hemoptysis hospitalizations in France. Chest 2000; 118: 440-444.

5 Hirshberg B, Biran I, Glazer M, et al. Hemoptysis: etiology, evaluation, and outcome in a tertiary referral hospital. Chest 1997; 112: 440-444.

6 Santiago S, Tobias J, Williams AJ. A reappraisal of the causes of hemoptysis. Arch Intern Med 1991; 151: 2449-2451.

7 Souders CR, Smith AT. The clinical significance of hemoptysis. N Engl J Med 1952; 247: 790-793.

8 Fidan A, Ozdoğan S, Oruç O, et al. Hemoptysis: a retrospective analysis of 108 cases. Respir Med 2002; 96 : 677-680.

9 Herth F, Ernst A, Becker HD. Long-term outcome and lung cancer incidence in patients with hemoptysis of unknown origin. Chest 2001; 120: 1592-1594.

10 Lorgis L, Cottenet J, Molins G, et al. Outcomes after acute myocardial infarction in HIV-infected patients: analysis of data from a French nationwide hospital medical information database. Circulation 2013; 127: 1767-1774.

11 Aboa-Eboulé C, Mengue D, Benzenine E, et al. How accurate is the reporting of stroke in hospital discharge data? A pilot validation study using a population-based stroke registry as control. J Neurol 2013; 260: 605-613.

12 Quantin C, Cottenet J, Vuagnat A, et al. Qualité des données périnatales issues du PMSI: comparaison avec l'état civil et l'enquête nationale périnatale 2010 [Quality of perinatal statistics from hospital discharge data: comparison with civil registration and the 2010 National Perinatal Survey]. J Gynecol Obstet Biol Reprod 2014; 43: 680-690. 
13 Hanf M, Quantin C, Farrington P, et al. Validation of the French national health insurance information system as a tool in vaccine safety assessment: application to febrile convulsions after pediatric measles/mumps/rubella immunization. Vaccine 2013; 31: 5856-5862.

14 Gusmano M, Rodwin V, Weisz D, et al. Comparison of rehospitalization rates in France and the United States. J Health Serv Res Policy 2015; 20: 18-25.

15 Quantin C, Benzenine E, Velten M, et al. Self-controlled case series and misclassification bias induced by case selection from administrative hospital databases: application to febrile convulsions in pediatric vaccine pharmacoepidemiology. Am J Epidemiol 2013; 178: 1731-1739.

16 Lainay C, Benzenine E, Durier J, et al. Hospitalization within the first year after stroke: the Dijon Stroke Registry. Stroke 2015; 46: 190-196.

17 Pan W-H, Li L-A, Tsai M-J. Temperature extremes and mortality from coronary heart disease and cerebral infarction in elderly Chinese. Lancet 1995; 345: 353-355.

18 Yan YY. The influence of weather on human mortality in Hong Kong. Soc Sci Med 2000; 50: $419-427$.

19 Rothwell PM, Wroe SJ, Slattery J, et al. Is stroke incidence related to season or temperature? The Oxfordshire Community Stroke Project. Lancet 1996; 347: 934-936.

20 Lejeune JP, Vinchon M, Amouyel P, et al. Association of occurrence of aneurysmal bleeding with meteorologic variations in the north of France. Stroke 1994; 25: 338-341.

21 Fartoukh M, Khoshnood B, Parrot A, et al. Early prediction of in-hospital mortality of patients with hemoptysis: an approach to defining severe hemoptysis. Respiration 2012; 83: 106-114.

22 Delage A, Tillie-Leblond I, Cavestri B, et al. Cryptogenic hemoptysis in chronic obstructive pulmonary disease: characteristics and outcome. Respiration 2010; 80: 387-392.

23 Douglass BE, Carr DT. Prognosis in idiopathic hemoptysis. J Am Med Assoc 1952; 150: 764-765.

24 Samama MM, Conard J, Lillo-Le Louët A. Accidents hémorragiques des nouveaux anticoagulants oraux et examens de la coagulation [Hemorrhagic accidents of the new oral anticoagulants and coagulation assays]. $J \mathrm{Mal}$ Vasc 2013; 38: 259-270.

25 Halperin JL, Hankey GJ, Wojdyla DM, et al. Efficacy and safety of rivaroxaban compared with warfarin among elderly patients with nonvalvular atrial fibrillation in the Rivaroxaban Once Daily, Oral, Direct Factor Xa Inhibition Compared with Vitamin K Antagonism for Prevention of Stroke and Embolism Trial in Atrial Fibrillation (ROCKET AF). Circulation 2014; 130: 138-146.

26 Garcia-Olivé I, Sanz-Santos J, Centeno C, et al. Results of bronchial artery embolization for the treatment of hemoptysis caused by neoplasm. J Vasc Interv Radiol 2014; 25: 221-228.

27 Chen J, Chen L-A, Liang Z-X, et al. Immediate and long-term results of bronchial artery embolization for hemoptysis due to benign versus malignant pulmonary diseases. Am J Med Sci 2014; 348: 204-209.

28 Virally J, Choudat L, Chebbo M, et al. Épidémiologie et délais de prise en charge de 355 patients atteints de cancer bronchique [Epidemiology and delays in the management of 355 patients with lung cancer]. Rev Mal Respir 2006; 23: 43-48.

29 Leveque N, Brouchet L, Lepage B, et al. Analyse des délais de prise en charge des cancers thoraciques: étude prospective [An analysis of delays in the management of thoracic cancers: a prospective study]. Rev Mal Respir 2014; 31: 208-213. 